P. Feit

Nagoya Math. J.

Vol. 133 (1994), 177-187

\title{
EXPLICIT FORMULAS FOR LOCAL FACTORS: ADDENDA AND ERRATA
}

\author{
PAUL FEIT
}

\section{Introduction}

In [3], the author studied certain local integrals derived from Fourier coefficient computations on Eisenstein series. Members of a family of Dirichlet series were characterized as a product of an explicit term with a mysterious polynomial factor. In a recent letter to the author, Professor Shoyu Nagaoka asked specific questions concerning the polynomial factor. Several of these questions can be answered by the techniques in [3]. In Part I of that paper, the relevant term is described precisely; however, in Part II, the term is described as a mysterious, albeit finite, sum. The present paper complete [3] by recording what little is known of that sum.

We illustrate our tables by settling one of the questions raised in Professor Nagaoka's letter. Let $F$ is a totally real number field and let $K / F$ be a purely imaginary quadratic extension. Let $\mathscr{D}$ be the discriminant of $K / F$, and let $h \in \mathscr{D}^{-1}$. For a finite prime $\mathscr{P}$ of $F$,

$$
\bar{\alpha}_{\mathscr{P}}^{(2)}\left(S,\left[\begin{array}{ll}
h & 0 \\
0 & 0
\end{array}\right]\right)=\left(1-q^{-s}\right)\left(1-\phi(\mathscr{P}) q^{1-s}\right)\left(1-\phi(\mathscr{P}) q^{2-s}\right)^{-1}\left(\sum_{j=0}^{b} q^{j(3-s)}\right),
$$

where the $\alpha$-series derives from Eisenstein series for the hermitian modular group of genus $2, \phi$ is the ideal character of $K / F$ (normalized to be 0 if $\mathscr{P}$ ramifies), $q=N \mathscr{P}$, and $\mathscr{P}^{b}$ devides the ideal $(h) \mathscr{D}$ while $\mathscr{P}^{b+1}$ does not.

\section{The $\alpha$-series}

Let $F$ be a local of any characteristic except 2 and let $R$ be (a choice of) the ring of integers of $F$. Let $\mathscr{P}$ be the prime of $R$, and put

$$
q=N \mathscr{P} \text {. }
$$

Received January 19, 1993. 
Let $A$ be a semi-simple finite dimensional $F$-algebra, and let $B$ be the corresponding maximal order of $A$. For $k \in \mathbf{N}$, let $B^{k}$ be the right $B$-module of $k \times 1$ column vectors. For $k, r \in \mathbf{N}$ such that $k \geq r$, an $r \times k$ matrix $M$ with entries in $B$ is called primitive if there is a $(k-r) \times k$ matrix $N$ such that

$$
\left(\begin{array}{c}
N \\
M
\end{array}\right) \in G L_{k}(B)
$$

If $L$ is a $B$-module and $K \subseteq L$ is a submodule, let $[L: K]$ be the cardinality of $|L / K|$. If $L$ and $K$ are $B$-submodules of $A^{k}$ for some $k \in \mathbf{N}$, then define $[L: K]=[L: L \cap K] /[K: L \cap K]$ if each index on the right is finite. For $k \in \mathbf{N}$, define $v: G L_{k}(A) \rightarrow \mathbf{Q}$ by

$$
q^{v(T)}=\left[B^{k}: T \cdot B^{k}\right] .
$$

In practice, the function $q^{v(T)}$ is $|d t(T)|_{\mathscr{P}}^{-d}$, where $d t$ is some sort of reduced norm function $G L_{k}(A) \rightarrow F$, $\|_{\mathscr{P}}$ is a normalized valuation at $\mathscr{P}$ and $d$ is a positive constant. In [3] and in what follows, we work with the function $v$ instead of determinants and valuations. For this reason, our $\alpha$-series differ by a constant exponent from the usual ones, as used in [1] or [4]. We will comment on this later.

Let $k \in \mathbf{N}$. For $T \in M_{k}(A)$, define $j(T)$ by

$$
q^{j(T)}=\left[T B^{k}+B^{k}: B^{k}\right] .
$$

Another interpretation for $j(T)$ is as follows. Express $T=D^{-1} C$ where $(C D)$ is a primitive $k \times(\dot{2} k)$ matrix. Then $j(T)=v(D)$. Again, in other treatments, the $j$-factor is typically replaced by $|d t(D)|_{\mathscr{P}}^{-1}$.

Fix a non-trivial group character $\chi$ from the additive group of $F$ to the unit circle of $\mathbf{C}$. For our present purposes, any character will do. When we refer to Professor Nagaoka's question, we adopt the standard choice. Extend $\chi$ to $M_{k}(A)$ for each $k \in \mathbf{N}$ by composing the original character with the reduced trace, as described in [3].

Let $\rho$ be an involution for $A / F$. Let $U(\rho)$ be the set of $B$-units $\varepsilon$ such that $\varepsilon \varepsilon^{\rho}=1$. For $\varepsilon \in U$, a $(\rho, \varepsilon)$-hermitian lattice is a free $B$-module $M$ of finite rank paired with an $R$-bilinear form (,) $: M \times M \rightarrow A$ such that for $x, y \in M$ and $b, c \in B$.

$$
\begin{aligned}
& (b x, c y)=b \cdot(x, y) \cdot c^{\rho}, \\
& (x, y)=\varepsilon(y, x)^{\rho} .
\end{aligned}
$$

Let $k \in \mathbf{N}$. For each $\varepsilon \in U$, put 


$$
\begin{aligned}
& \sum(k, \varepsilon)=\left\{T \in M_{k}(A): T=\varepsilon\left({ }^{t} T^{o}\right)\right\} \\
& \sum(k, \varepsilon, B)=\sum(k, \varepsilon) \cap M_{k}(B),
\end{aligned}
$$

and also

$$
\sum(k, \varepsilon, B) \#=\left\{T \in \sum\left(k, \varepsilon^{o}\right): \chi\left(T \cdot \sum(k, \varepsilon, B)\right)=\{1\}\right\} .
$$

The lattice $\sum(k, \varepsilon)$ obviously corresponds to all $(\rho, \varepsilon)$-hermitian forms on $B^{k}$. We refer to its members as being $(\rho, \varepsilon)$-hermitian. The function which takes $T \in \sum(k, \varepsilon, B) \#$ to the function $X \rightarrow \chi(T X)$ identifies the additive group of $\sum(k, \varepsilon, B)$ \# with the character group of $\sum(k, \varepsilon, B)$; for that reason, we refer to the former as the dual lattice.

Because most of the work in [3] deals with dual lattices, we set the problem in a manner in which the members of the dual lattice are $(\rho, \varepsilon)$-hermitian. For this reason, we set up the $\alpha$-series as a sum over $\sum\left(k, \varepsilon^{o}\right)$ instead of $\sum(k, \varepsilon)$.

Let $\rho$ be an involution of $A$, let $\varepsilon \in U(\rho)$, let $m \in \mathbf{N}$ and let $N \in \sum\left(m, \varepsilon^{o}\right.$, $B)$ \# . Define the $\alpha$-series for this data by

$$
\alpha(N, t)=\sum_{x \in \Sigma\left(m, \varepsilon^{\rho}\right) / \Sigma\left(m, \varepsilon^{\rho}, B\right)} \chi(N x) \cdot t^{j(x)},
$$

where $t$ is a formal variable. This is the correct form of [3; (5.10)], with $B$ playing the role of $S$. The Dirichlet $\alpha$-series used by Nagaoka [1] or Shimura [4] have the form

$$
\alpha(N, s)=\alpha\left(N, q^{-s / d}\right),
$$

where the constant $d$ is the exponent factor characterized by $q^{v(T)}=|d t(T)|_{\mathscr{P}}{ }^{-d}$ Tautologically, for any $u \in G L_{k}(B), \alpha\left(u N \cdot{ }^{t} u^{o}, t\right)=\alpha(N, t)$

Analysis of the $\alpha$-series divides into two cases. First, suppose $A=\Delta \oplus \Delta^{\circ}$, where $\Delta$ is a simple $F$-algebra and $\Delta^{\circ}$ is its opposite, and $\rho$ is defined by $(b, c) \rightarrow$ $(c, b)$. In this case, the involution $\rho$ and the choice of $\varepsilon$ is irrelevant. The $\alpha$-series (8) can be rephrased as an infinite sum over $M_{k}(\Delta)$. The reformulation is analyzed in [3; Part I]. The analysis is complete, and we will make no additions to it here.

\section{Hermitian lattices}

With the split case settled, all other situations reduce to the hypothesis

$A$ is a division $F$-algebra,

$F$ is the fixed field of $\rho$ on the center of $A$. 
Under assumption (10), we hereafter denote $A$ by $\Delta$ and the ring $B$ by $S$. Fix $\varepsilon \in U(\rho)$. From now on, for $T$ a square matrix, put

$$
\begin{aligned}
T^{*} & ={ }^{t} T^{\rho}, \quad \text { and } \\
T^{-*} & =\left(T^{*}\right)^{-1} \quad \text { if } T \text { is invertible. }
\end{aligned}
$$

For $k \in \mathbf{N}, N \in M_{k}(\Delta)$ and $C \in G L_{k}(\Delta)$, put $N[C]=C^{-1} N C^{-*}$.

Let $\mathbf{m}$ be the maximal ideal of $S$, and let $\pi$ be a generator of $\mathbf{m}$. Define a logarithmic valuation $\mathfrak{l}$ on $\Delta^{*}=\Delta-\{0\}$ by

$$
\forall x \in \Delta^{*}, \pi^{-l(x)} x \in S-\mathbf{m} .
$$

We adopt the convention that $\mathfrak{l}(0)=\infty$. For $X \subseteq \Delta$ a non-empty set, put

$$
\mathfrak{l}(X)=\inf \{\mathfrak{l}(x): x \in X\} .
$$

For $M$ a hermitian lattice, define

$$
s(M)=\mathfrak{l}(\{(x, y): x, y \in M\}) .
$$

For $n \in \mathbf{Z}$, put

$$
\begin{aligned}
& \Delta_{n}=\{d \in \Delta: l(d) \geq n\}, \\
& A_{n}=\left\{b+\varepsilon b^{\rho}: b \in \Delta_{n}\right\} .
\end{aligned}
$$

Put

$$
\begin{aligned}
& \mathscr{D}=\left\{d \in \Delta: \forall b \in S, \chi\left(b d+b^{\rho} d^{\rho}\right)=1\right\}, \\
& \delta=\mathfrak{l}(\mathscr{D}) .
\end{aligned}
$$

For $n \in \mathbf{Z}$, let Cat $(\rho, \varepsilon, n)$ be the class of all $(\rho, \varepsilon)$-hermitian lattices $M$ such that

$$
\begin{aligned}
& s(M) \geq n, \\
& \forall v \in M, \quad(v, v) \in A_{n} .
\end{aligned}
$$

In [3; Section 8], we define a notion of morphism between members of Cat $(\rho, \varepsilon, n)$, and turn the class into a category. That structure is technical, and is omitted here. Certain lattices in this category have a special property, and are called $n$-modular; again, the precise definition is omitted, and we refer the reader to [3] for proof of the properties of $n$-modular lattices which we need. The hyperbolic lattices of denominator $-n$ are $n$-modular.

Parameters $\sigma, \sigma_{1}, \sigma_{2}, \sigma_{3}, \sigma_{4}$ and $\sigma_{5}$ are defined in [3; (5.8) and (5.9)]. Except for $\sigma_{2}$, these are usually trivial to calculate. To get $\sigma_{2}$, use the fact [3; Lemma 5.1] 


$$
\sigma_{1}+\sigma_{2}+\sigma_{3}=\sigma_{4}+\sigma
$$

Depending on these parameters and on $n$, the category Cat $(\rho, \varepsilon, n)$ is classified as one of four types, in [3; (8.19)]. The category relevant to our calculation is Cat $(\rho$, $\varepsilon, \delta)$. It is also a consequence of [3; Lemma 5.1] that, for $k \in \mathbf{N}, \sum\left(k, \varepsilon^{o}, S\right) \#$ is the set of all matrices which correspond to member of $\operatorname{Cat}(\rho, \varepsilon, \delta)$ of rank $k$.

The function $v_{1}$, on square, invertible matrices, is introduced in [3; Definition 7.1]. The only comments that we make here are (a) $v_{1}$ depends on $\rho$ and $\varepsilon$, and (b) like $v, v_{1}(T)$ has the form $|d t(T)|_{\mathscr{P}}{ }^{-d_{1}}$ where $d_{1}$ is some constant dependent on the raw data.

\section{Definite exponents}

For the next part of the argument, fix $m \in \mathbf{N}$. Fix $N \in \sum\left(m, \varepsilon^{o}, S\right) \# \cap$ $G L_{m}(\Delta)$. Express

$$
m=2 g_{0}+\lambda_{0} \text {, where } g_{0} \in \mathbf{Z} \text { and } \lambda_{0} \in\{0,1\} .
$$

We now add a parameter not in [3]. Depending on the type of Cat $(\rho, \varepsilon, \delta)$, define $\lambda_{1}$ as

$$
\lambda_{1}= \begin{cases}\lambda_{0} & \text { for Type I, } \\ 0 & \text { for Type II or IV } \\ 1 & \text { for Type III. }\end{cases}
$$

Let

$$
Y(N)=\left\{C \in G L_{m}(\Delta) \cap M_{m}(S): N[C] \in \sum\left(m, \varepsilon^{o}, S\right) \#\right\} .
$$

Note that $G L_{m}(S)$ acts on $Y$ on the right, and the quotient $Y(N) / G L_{m}(S)$ is finite. Following Siegel, our first major result is that $\alpha(N, t)$ is a sum of terms, one for each $C \in Y / G L_{m}(S)$. The term for $C$ has to do with the structure of $N[C]$ in Cat $(\rho, \varepsilon, \delta)$.

For

$$
g, h \in \mathbf{N} \cup\{0\}, \lambda, \mu \in\{0,1\} \text { and } \eta \in\{-1,1\} \text {, }
$$

define a polynomial in the indeterminate $t$ by

$$
\begin{aligned}
& R(g, h, \lambda, \eta, \mu ; t)=\prod_{j=0}^{g+h+\lambda-1}\left(1-q^{j \sigma_{3}} t^{\sigma_{3}}\right) \\
& \times\left\{\left(1+\eta(1-\mu) q^{(g+h) \sigma_{3}+\sigma_{1}+\sigma_{2}-\sigma} t^{\sigma_{3}}\right) \prod_{i=1}^{g+h-1}\left(1+q^{\imath \sigma_{3}+\sigma_{1}+\sigma_{2}-\sigma} t^{\sigma_{3}}\right)\right\},
\end{aligned}
$$


where the bracketed part is set equal to 1 if $g+h=0$. Equation (22) is the correct form of (9.22) in [3]. We only consider this function when $\mu \leq h, \eta=1$ if $\lambda=1$, and $\lambda=0$ if $\mu=1$.

The significance of (22) is as follows. Let $M \in \sum\left(m, \varepsilon^{\rho}, S\right) \# \cap G L_{m}(\Delta)$. Regard $M$ as a hermitian structure on $S^{m}$. Then $M$ is isomorphic to an orthogonal sum $L \perp D$ where $L$ is $\delta$-modular and $s(D)>\delta$. Define $(g, h, \lambda, \eta, \mu)=(g(M)$, $h(M), \lambda(M), \eta(M), \mu(M))$ to be the unique tuple which satisfies (21) and

$$
\begin{aligned}
& \operatorname{rank}(L)=2 \mathrm{~g}+\lambda, \\
& \operatorname{rank}(D)=h \\
& \eta=-1 \text { if and only if } L \text { has even rank and is not hyperbolic, } \\
& \mu \text { is the defect of } D .
\end{aligned}
$$

The defect is defined in [3; Definition 8.3], and generalizes the classical notion of defect in quadratic forms over fields of characteristic 2. It occurs only for Type IV situations. Define

$$
R(M ; t)=R(g(M), h(M), \lambda(M), \eta(M), \mu(M) ; t) .
$$

Now for $C \in Y(N) / G L_{m}(S)$, put $R(N, C ; t)=R(N[C] ; t)$. Then

$$
\alpha(N, t)=\sum_{C \in Y(N) / G L_{m}(S)} q^{(r-1) v(C)+v_{1}(C)} t^{2 v(C)} R(N, C ; t) .
$$

We shall isolate the greatest common divisor of the summands in (25).

If there is $C \in Y(N)$ such that $N[C]$ is modular, define $\eta_{0}=\eta_{0}(N)$ to be 1 unless $N[C]$ has even rank and is not hyperbolic; in the latter case, define $\eta_{0}=$ - 1. If $N[C]$ is not modular for any $C$, put $\eta_{0}=0$. For each $\mathrm{C} \in Y(N)$, define

$$
\begin{aligned}
& P(N, C ; t)=q^{(r-1) v(C)+v_{1}(C)} t^{2 \cdot v(C)} \prod_{j=g_{0}+\lambda_{0}}^{g+h+\lambda-1}\left(1-q^{j \sigma_{3}} t^{\sigma_{3}}\right) \\
& \times\left\{\frac{\left(1+\eta(1-\mu) q^{(g+h-1) \sigma_{3}+\sigma_{4}} t^{\sigma_{3}}\right)}{\left(1+\eta_{0} q^{\left(g_{0}-1\right) \sigma_{3}+\sigma_{4}} t^{\sigma_{3}}\right)}\right\} \times \prod_{i=g_{0}-1}^{g+h-2}\left(1+q^{i \sigma_{3}+\sigma_{4}} t^{\sigma 3}\right) \text { if } \eta_{0} \neq 0 \text {, or }
\end{aligned}
$$

$$
\begin{aligned}
& P(N, C ; t)=q^{(\gamma-1) v(C)+v_{1}(C)} t^{2 \cdot v(C)} \prod_{j=g_{0}+1}^{g+h+\lambda-1}\left(1-q^{j \sigma_{3}} t^{\sigma_{3}}\right) \\
& \times\left\{\left(1+\eta(1-\mu) q^{(g+h-1) \sigma_{3}+\sigma_{4}} t^{\sigma_{3}}\right) \prod_{i=g_{0}+\lambda_{1}}^{g+h-2}\left(1+q^{i \sigma_{3}+\sigma_{4}} t^{\sigma 3}\right)\right\} \text { if } \eta_{0}=0,
\end{aligned}
$$

where, in the second formula, the bracketed expression is 1 if $g+h-1<g_{0}+$ $\lambda_{1}$. In fact, $P(N, C ; t)$ is $R(N, C ; t)$ divided by the greatest common factor of all polynomials $R\left(N, C^{\prime} ; t\right)$. Define $P(N ; t)$ be the sum of $P(N, C ; t)$ as $C$ 
varies over $Y(N) / G L_{m}(S)$. Essentially, $P(N ; t)$ is the troublesome generalization of the $\sigma$-functions that appear in the Eisenstein series for $S L_{2}(\mathbf{Q})$.

\section{Hermitian matrices of all ranks}

Suppose $N_{1} \in \sum\left(m, \varepsilon^{\rho}, S\right) \#$ has the form

$$
N_{1}=\left[\begin{array}{rr}
N & 0 \\
0 & 0
\end{array}\right]
$$

where $r \in \mathbf{N}, N \in \sum\left(r, \varepsilon^{o}, S\right) \# \cap G L_{r}(\Delta)$. If $N_{1}=0$, adopt the convention that $r=0$ and $\alpha(N, t)=1$; all of the formulas that follow will then be valid. Now

$$
a\left(N_{1}, t\right)=F_{m, r}(t) \cdot \alpha\left(N, q^{m-r} t\right)
$$

where

$$
F_{m, r}(t)=\frac{\prod_{i=0}^{m-r-1}\left(1+q^{i \sigma_{3}+\sigma_{4}} t^{\sigma 3}\right) \prod_{i=0}^{m-r-1}\left(1-q^{i \sigma_{3}} t^{\sigma 3}\right)}{\prod_{j=0}^{m-r-1}\left(1-q^{(m-1+j) \sigma_{3}+\sigma_{5}} t^{2 \sigma_{3}}\right)} .
$$

Define $g_{0}, \lambda_{0}, \lambda_{1}$ and $\eta_{0}$ as in the previous section, for the matrix $N$. (lf $r=0$, put $g_{0}=\lambda_{0}=\lambda_{1}=0$ and $\eta_{0}=1$.) Then $\alpha\left(N_{1}, t\right)$ is the product of $P\left(N, q^{m-r} t\right)$ times

$$
\begin{aligned}
& \left\{\frac{\prod_{i=0}^{m-g_{0}-\lambda_{0}-2}\left(1+q^{i \sigma_{3}+\sigma_{4}} t^{\sigma 3}\right) \prod_{i=0}^{m-g_{0}-1}\left(1-q^{i \sigma_{3}} t^{\sigma 3}\right)}{\prod_{j=0}^{m-r-1}\left(1-q^{(m-1+j) \sigma_{3}+\sigma_{5}} t^{2 \sigma_{3}}\right)}\right\} \\
& \times\left(1+\eta_{0} q^{\left(m-g_{0}-\lambda_{0}-1\right) \sigma_{3}+\sigma_{4}} t^{\sigma_{3}}\right) \text { if } \eta_{0} \neq 0 \text { and } g_{0} \neq 0,
\end{aligned}
$$

$$
\begin{gathered}
\left\{\frac{\prod_{i=0}^{m-\lambda_{0}-1}\left(1+q^{i \sigma_{3}+\sigma_{4}} t^{\sigma 3}\right) \prod_{i=0}^{m-1}\left(1-q^{i \sigma_{3}} t^{\sigma_{3}}\right)}{\prod_{j=0}^{m-r-1}\left(1+q^{(m-1+j) \sigma_{3}+\sigma_{5}} t^{2 \sigma_{3}}\right)}\right\} \text { if } \eta_{0} \neq 0 \text { and } g_{0}=0, \\
\left\{\frac{\prod_{i=0}^{m-g_{0}-\lambda_{0}+\lambda_{1}-1}\left(1+q^{i \sigma_{3}+\sigma_{4}} t^{\sigma_{3}}\right) \prod_{i=0}^{m-g_{0}-\lambda_{0}}\left(1-q^{i \sigma_{3}} t^{\sigma_{3}}\right)}{\prod_{j=0}^{m-r-1}\left(1+q^{(m-1+j) \sigma_{3}+\sigma_{5}} t^{2 \sigma_{3}}\right)}\right\} \text { if } \eta_{0}=0 .
\end{gathered}
$$

Table (30) is the correct form of [3; Theorem 5.3]. 


\section{On a question by Professor Nagaoka}

Let $F_{0}$ be a totally real number field, let $K_{0} / F_{0}$ be a purely imaginary quadratic extension field and let $\rho_{0}$ be the Galois involution of $K_{0} / F_{0}$. Let $\phi$ be the ideal character of $K_{0} / F_{0}$. Let $\mathscr{P}$ be a finite prime of $F_{0}$, let $F$ be the localization of $F_{0}$ at $\mathscr{P}$, and let $K=K_{0} \otimes_{F_{0}} F$ and $\rho=\rho_{0} \otimes_{F_{0}} 1_{F}$. Let $\omega$ be a local generator of $\mathscr{P}$, and put $q=N \mathscr{P}$. To normalize our series, we need to compare $v(\omega)$ with $|\omega|_{\mathscr{P}}^{-1}=q$.

Let $p$ be the rational prime which divides $q$, and let $\delta$ be a generator of the discriminant of $F / \mathbf{Q}_{p}$. On $\mathbf{Q}_{p}$, define $\chi_{0}$ by $\chi_{0}(t)=e^{2 \pi i r}$ for $r \in \mathbf{Q}$ any rational such that $r+t \in \mathbf{Z}_{p}$. Define $\chi_{F}$ to be the composition of $\chi_{0}$ with the trace function of $F / \mathbf{Q}_{p}$. If $M$ is any square matrix over $K$ whose trace $t$ lies in $F$, define $\chi(M)=\chi_{F}(t)$.

Let $h$ be a non-zero member of the different of $F / \mathbf{Q}_{p}$ - that is, the fractional ideal generated by $\delta^{-1}$ and let $b \in \mathbf{N} \cup\{0\}$ such that $\omega^{b}$ divides $h \delta$ while $\omega^{b+1}$ does not. We claim that

$$
\bar{\alpha}_{\mathscr{P}}^{(2)}\left(s,\left[\begin{array}{ll}
h & 0 \\
0 & 0
\end{array}\right]\right)=\left(1-q^{-s}\right)\left(1-\phi(\mathscr{P}) q^{1-s}\right)\left(1-\phi(\mathscr{P}) q^{2-s}\right)^{-1}\left(\sum_{\jmath=0}^{b} q^{j(3-s)}\right),
$$

where the $\alpha$-series derives from Eisenstein series for the hermitian modular group of genus 2 as in [1] or [4]. Here, $m=2, r=1$ and $\varepsilon=\varepsilon^{o}=1$.

The justification of (31) depends on the behavior of $\mathscr{P}$ in $K_{0}$. Different factorizations for $\mathscr{P}$ in $S$ require different tables.

Case I: $\mathscr{P}$ splits.

This is the situation not discussed in the present addendum. Here, $K \cong F \oplus F$, and [3; Part I] applies. Inspection shows that $v(\omega)=1$, so

$$
\bar{\alpha}_{\mathscr{P}}^{(2)}\left(s,\left[\begin{array}{ll}
h & 0 \\
0 & 0
\end{array}\right]\right)=\alpha\left(\left[\begin{array}{ll}
h & 0 \\
0 & 0
\end{array}\right] \cdot q^{-s}\right) .
$$

Although the discriminant is not mentioned by name in [3; Part 1], it is referred to in its role as genarator of the fractional ideal

$$
I=\{s \in F: \chi(R \cdot s)=\{1\}\} .
$$

The indexing set for the polynomial $p(E, t)$ defined in $[3 ;(2.4)]$ for the $1 \times 1$ matrix $(\delta h)$ can be represented by $\left\{\omega^{j}\right\}_{j=0}$. Thus,

$$
p(\delta h, t)=\sum_{j=0}^{b} t^{j}
$$


Using [3; (2.6)] for parameters $k=r=2, m=1$ and $\sigma$ (as defined in [3; Theorem 2.1]) equal to 1 , we get

$$
\bar{\alpha}_{\mathscr{P}}^{(2)}\left(s,\left[\begin{array}{ll}
h & 0 \\
0 & 0
\end{array}\right]\right)=\left(1-q^{-s}\right)\left(1-q^{1-s}\right)\left(1-q^{2-s}\right)^{-1}\left(\sum_{j=0}^{b} q^{j(3-s)}\right) .
$$

Since $\phi(\mathscr{P})=1,(33)$ is $(31)$.

All remaining cases refer to the new tables. Let us make some general comments.

Hereafter, we assume $K$ is a field extension of $F$. Let $S$ be its ring of integers, and let $\delta_{K}$ be its discriminant as a $\mathbf{Q}_{p}$-extension. Let $\pi$ be a generator of the prime of $S$.

We begin with a minor issue of normalization. For $M$ a square matrix over $K$, define $\tau(M)$ to be the image of $M$ 's trace under the trace map of $K / F$. Now [3; Part II] consider matrix characters of the form $\zeta{ }^{\circ} \tau$. The character used in [1] or [4] is not $\chi_{F}{ }^{\circ} \tau$. Because the character is evaluated only on matrices whose trace is in $F$, there is no need to apply the trace of the extension $K / F$. However, we can describe this standard character as $\chi^{\prime} \circ \tau$ where $\chi^{\prime}(x)=\chi_{F}(x / 2)$ ! Thus, the series of [3; Part II] do emulate the standard local integrals.

As in Case I, the discriminant $\delta$ plays a role. Let $k \in \mathbf{N}$. The dual lattice $\sum\left(k, \varepsilon^{\rho}, S\right) \#$ consists of all $k \times k(\rho, 1)$-hermitian matrices whose diagonal entries lie in the fraction $F$-ideal generated by $\delta^{-1}$ and whose off-diagonal entries lie in the fractional $K$-ideal generated by $\delta_{K}^{-1}$. Again, we fix $h \in \delta^{-1} R$.

The key parameters specialize as

$$
\begin{array}{ll}
\sigma=2, \sigma_{1}=1, \sigma_{2}=0, \sigma_{3}=2, & \sigma_{4}=1, \sigma_{5}=2, \\
v(\pi)=2, v_{1}(\pi)=2 & \text { if } \mathscr{P} \text { is unramified, } \\
\sigma=2, \sigma_{1}=1, \sigma_{2}=0, \sigma_{3}=1, & \sigma_{4}=0, \sigma_{5}=1, \\
v(\pi)=1, v_{1}(\pi)=1 & \text { if } \mathscr{P} \text { ramifies. }
\end{array}
$$

The unramified situation will divide into two cases.

Regardless of ramification, $\sigma>\sigma_{1}+\sigma_{2}$. Thus, Cat $(\rho, 1, \delta)$ is of Type I or Type III. In particular, the defect of any hermitian lattice will be 0 . Classically, the defect is a concept related to quadratic forms rather than hermitian forms. Its present irrelevance is not surprising.

Regardless of ramification, $v(\omega)=2$. This means that we wish to replace the variable $t$ by $q^{-s / 2}$ to get the appropriate Dirichlet series. In general, the exponential constant factor will be $1 / \sigma$.

We generate the polynomial for the matrix $N=(h)$. In this calculation, 
$g_{0}=0$ and $\lambda_{0}=1$. The $\eta$ term for $N[C]$ will always be 1 , while $\eta_{0}$ could be 0 or 1 , depending on $h$.

Case II: $\mathscr{P}$ is unramified, $b=2 y$ is even.

The polynomial $P(N ; t)$ is a sum indexed by matrices $c=\left(\omega^{x}\right)$ for $0 \leq x$ $\leq y$. When $x=y, N[c]$ is modular, hence, $\eta_{0}=1$, and $P\left(N, \omega^{y} ; t\right)=q^{2 y} t^{4 y}$. For $0 \leq x<y$, the key parameters are $g=0, h=1, \lambda=0, \eta=1$ and $\mu=0$, which yields

$$
P\left(N, \omega^{x} ; t\right)=q^{2 x} t^{4 x}\left(1+q t^{2}\right)=q^{2 x} t^{4 x}+q^{2 x+1} t^{2(2 x+1)} .
$$

Consequently,

$$
\begin{aligned}
& P(N ; t)=\sum_{j=0}^{2 y} q^{j} t^{2 j} . \\
& P(N ; q t)=\sum_{j=0}^{2 y} q^{3 \jmath} t^{2 j}=\sum_{j=0}^{b}\left(q^{3} t^{2}\right)^{j} .
\end{aligned}
$$

The extra factor (30) works out to be

$$
\frac{\left(1+q t^{2}\right)\left(1-t^{2}\right)\left(1-q^{2} t^{2}\right)}{\left(1-q^{4} t^{4}\right)}=\frac{\left(1+q t^{2}\right)\left(1-t^{2}\right)}{\left(1+q^{2} t^{2}\right)} .
$$

Now replace $t$ by $q^{-s / 2}$ and combine the terms to get

$$
\left(1-q^{-s}\right)\left(1+q^{1-s}\right)\left(1+q^{2-s}\right)^{-1}\left\{\sum_{j=0}^{b} q^{j(3-s)}\right\} .
$$

This is exactly (31) after replacing $\phi(\mathscr{P})=-1$.

Case III: $\mathscr{P}$ unramified, $b=2 y+1$ is odd

In this case, $\eta_{0}=0$, and we use different formulas. Since the relevant category is Type I or Type III, the parameter $\lambda_{1}$ must be 1 . For $0 \leq x \leq y$, the parameters are $g=0, h=1, \lambda=0, \eta=1, \mu=0$, and

$$
P\left(N, \omega^{x} ; t\right)=q^{2 x} t^{4 x}
$$

The combined factor is

$$
\begin{gathered}
\frac{\left(1+q t^{2}\right)\left(1+q^{3} t^{2}\right)\left(1-t^{2}\right)\left(1-q^{2} t^{2}\right)}{\left(1-q^{4} t^{4}\right)}\left\{\sum_{j=0}^{y} q^{6 j} t^{4 j}\right\} \\
=\left\{\frac{\left(1+q t^{2}\right)\left(1-t^{2}\right)}{\left(1+q^{2} t^{2}\right)}\right\}\left\{\left(1+q^{3} t^{2}\right) \sum_{j=0}^{y} q^{6 j} t^{4 j}\right\}
\end{gathered}
$$




$$
=\left\{\frac{\left(1+q t^{2}\right)\left(1-t^{2}\right)}{\left(1+q^{2} t^{2}\right)}\right\}\left\{\sum_{j=0}^{2 y+1} q^{3 j} t^{2 j}\right\} .
$$

Again after substitution $t=q^{-s / 2}$, we get (31) with $\phi(\mathscr{P})=-1$.

Case IV: $\mathscr{P}$ is ramified

We may choose that $\omega=\pi \pi^{\rho}$. Since $\mathscr{P}$ is ramified, Cat $(\rho, 1, \delta)$ is Type III. Thus, $g_{0}=0, \lambda_{0}=1$ and $\eta_{0}=0$. For each $0 \leq x \leq b, P\left(N, \pi^{x} ; t\right)$ has parameters $g=0, h=1, \lambda=0, \eta=1$ and $\mu=0$. We get

$$
P(N ; t)=\sum_{j=0}^{b} q^{j} t^{2 j} \text {, and } P(N ; q t) \underset{j=0}{\stackrel{b}{=}} q^{3 j} t^{2 j} .
$$

Happily, the extra factor (30) simplifies:

$$
\frac{(1+t)(1+q t)(1-t)(1-q t)}{\left(1-q^{2} t^{2}\right)}=\left(1-t^{2}\right) .
$$

The net rational factor becomes

$$
\left(1-t^{2}\right) \sum_{j=0}^{b} q^{3 j} t^{2 j}
$$

After substitution $t=q^{-s / 2}$, we get $(31)$ with $\phi(\mathscr{P})=0$.

\section{REFERENCES}

[1] S. Nagaoka, On the Fourier coefficient of Hermitian Eisenstein series of degree 2, preprint.

[2] P. Feit, Poles and residues of Eisenstein series for sympletic and unitary groups, Memoirs of the Amer. Math. Soc., 346, 1986.

[3] - Explicit formulas for local factors in the Euler products for Eisenstein series, Nagoya Math. J., 113 (1989), 37-87.

[4] G. Shimura, On Eisenstein series, Duke Math. J., 50 (1983), 417-176.

University of Texas at Permian Basin

Science and Engineering

4901 F. University Blvd.

Odessa, TX 79762, U.S.A. 